\title{
Remarks on Extremal Overfull Graphs
}

\author{
Modjtaba Ghorbani \\ Department of Mathematics, Faculty of Science, Shahid Rajaee Teacher Training University, Tehran, Iran \\ Email: mghorbani@srttu.edu
}

Received May 23, 2013; revised June 23, 2013; accepted July 1, 2013

Copyright @ 2013 Modjtaba Ghorbani. This is an open access article distributed under the Creative Commons Attribution License, which permits unrestricted use, distribution, and reproduction in any medium, provided the original work is properly cited.

\begin{abstract}
An overfull graph is a graph whose number of its edges is greater than the product of its maximum degree and $[n / 2]$, where $n$ is the number of vertices. In this paper, some extremals of overfull graphs are presented. We also classify all plannar overfull graphs.
\end{abstract}

Keywords: Overfull Graph; Edge Chromatic Number; Plannar Graph

\section{Introduction}

All graphs in this paper are simple and denoted by $G=(V, E)$. The $k$-edge coloring of a graph is an assignment of $k$ colors to the edges of the graph so that adjacent edges have different colors. The minimum required number of colors for the edges of a given graph is called the edge chromatic number of the graph and it is denoted by $\chi^{\prime}(G)$. In the next section, we compute some extremal overfull graphs and finally, in section three, we determinethe class of plannar overfull graph. Throughout this paper, our notation is standard and mainly taken from [1].

\section{Results and Discussion}

Let $\Delta(G)$ be the maximum degree of vertices of graph $G$. Obviously, $\chi^{\prime}(G) \geq \Delta(G)$, and by Vizing's theorem $\chi^{\prime}(G) \leq \Delta(G)+1$. In other words, $\chi^{\prime}(G)=\Delta(G)$ or $\chi^{\prime}(G)=\Delta(G)+1$. The graph $G$ is said to be of class 1 whenever, $\chi^{\prime}(G)=\Delta(G)$ and otherwise, it is said to be of class 2 .

Let $G$ be a graph with $n$ vertices and $m$ edges, then $G$ is overfull graph if $m>\Delta(G) \cdot[n / 2]$. It is easy to see that the number of vertices of an overfull graph is an odd number and they are class 2 . The following lemma, directly can be derived from the definition:

Lemma 1. Every $r$-regular graph is overfull, where $r$ is an even and $n$ is an odd integers.

The concept of overfull graph play a significant role in understanding of the edge chromatic properties of graphs. Chetwynd and Hilton [2] conjectured that a vaste category of graphs are class 2 if they contain an overfull subgraph with the same maximum degree:

Conjecture (Overfull Conjecture). A graph $G$ with $\Delta(G)>|V(G)| / 3$ is class 2 if and only if it contains an overfull subgraph $H$ such that $\Delta(G)=\Delta(H)$.

We know that this conjecture is solved under special conditions (see e.g. [3,4]).

The aim of this section is to compute the maximal and minimal overfull graphs. We show that trees and unicycle graphs are not overfull. In continuing, we compute the second, the third and the fourth extremal overfull graphs. Throughout this section suppose $G$ is a graph with $n$ vertices and $m$ edges, where $n$ is an odd integer. Let $e=u v$ be an edge of $G$ and $G+e$ be a graph obtained from $G$ by adding $e$. If $G+e$ be again an overfull graph, then $e$ is not a pendant edge, since the number of vertices of an overfull graph is an integer. Further, we have the following lemma:

Lemma 2. Let $G$ be a connected graph with an odd $n \geq 3$ vertices. If $G$ has a pendent edge, then $G$ is not overfull.

Proof. Suppose $G$ has a pendent vertex and $\Delta=n-1$. So, the maximum number of edges is

$$
m=\frac{(n-1)(n-2)}{2}+1<\frac{(n-1)}{2}(n-1)=\frac{n-1}{2} \Delta .
$$

So, $G$ is not overfull. Similarly, one can see that in other cases $G$ is not overfull.

Lemma 3. If $G$ be a unicycle overfull graph, then $G$ is a cycle.

Proof. Let $G$ be a unicycle overfull graph, thus $n>\Delta \frac{n-1}{2}$ and so $n<\frac{\Delta}{\Delta-2}$. Since $n \geq 3$, hence 
$\frac{\Delta}{\Delta-2} \geq 3$ and then $\Delta \leq 3$.

- If $\Delta=3$ then $m=n>\frac{3}{2}(n-1)$ if and only if $n<3$, a contradiction.

- If $\Delta=2$ then $G \cong C_{n}$ and the proof is completed.

An overfull graph is minimal if it has the minimum number of edges among all $n$ vertices overfull graphs and it is maximal if it has the maximum number of edges. In the following theorem we find the minimal and maximal overfull graphs:

Theorem 1. Let $n \geq 3$, then among all $n$ vertices overfull graphs, the complete graph $K_{n}$ is maximal and the cycle $C_{n}$ is minimal.

Proof. Let $n \geq 3$, the first claim is clear. For the second, since $G$ is overfull then $\Delta>2$ and so, $m>n-1$. This implies that $G$ has a cycle. Clearly, $G$ is minimal overfull graph if and only if $m=n$. By using Lemma $3, \Delta=2$ and so $G$ is a cycle.

In Lemma 3, we classified the unicycle graphs on $m=n$ edges. In continuing, let $G$ be a graph with $m=n+1$ edges, since $G$ is overfull, thus

$$
\begin{aligned}
& n+1>\Delta \frac{n-1}{2} \Leftrightarrow 2 n+2>\Delta n-\Delta \\
& \Leftrightarrow(\Delta-2) n<\Delta+2 \Leftrightarrow n<\frac{\Delta+2}{\Delta-2} .
\end{aligned}
$$

But $n \geq 3$ implies that $\frac{\Delta+2}{\Delta-2}>3$ and hence $\Delta<4$.

- If $\Delta=2$ then $G$ is a graph on $n$ vertices with $n+1$ edges, a contradiction.

- If $\Delta=3$ then $n+1>\frac{3}{2}(n-1)$ if and only if $n<5$, a contradiction.

Therefore we proved the following theorem:

Theorem 2. Let $G$ be a graph on $n$ vertices and $m=n+1$ edges, then $G$ is not overfull.

As a result of the last theorem one can see that the second minimal overfull graph is not belong to the class of $\theta$-graphs.

Let $f$ be an arbitrary edge of a cycle $C_{n}$ on $n$ vertices. Add $\frac{n-3}{2}$ new edges to $C_{n}$, parallel with $f$ and then join an endpoint of $f$ to the remained vertex of degree 2, the resulted graph is an overfull graph and we denote it by $C_{n}+\frac{n-1}{2} e$.

Here, we determine the second extremal overfull graph. Let us consider graphs with $n$ vertices and $n+2$ edges. It is easy to see that

$$
n+2>\Delta \frac{n-1}{2} \Leftrightarrow 2 n+4>\Delta n-\Delta \Leftrightarrow n<\frac{\Delta+4}{\Delta-2} .
$$

Since $n \geq 3$, so $\Delta<5$ and we have the following cases:

- If $\Delta=4$, then $n+2>2(n-1)$ if and only if $2 n-n<4$ if and only if $n<4$, a contradiction.

- If $\Delta=3$, then $n+2>\frac{3}{2}(n-1)$ if and only if $2 n+4<3 n-3$, if and only if $n<7$. Clearly, $n=5$ and in this case, $G$ is overfull graph isomorphic with $C_{5}+2 e$. So, we proved the following theorem:

Theorem 3. Among all graphs on $n$ vertices and $m=n+2$ edges, only $C_{5}+2 e$ is overfull.

Let now $G$ be a graph with $n$ vertices and $m=n+3$ edges. By a similar way with Theorem 2, one can see that $n+3>\Delta \frac{n-1}{2}$ if and only if $n<\frac{\Delta+6}{\Delta-2}$. Since $n \geq 3$ thus $\Delta<6$ and so we have three following cases:

- If $\Delta=5$, then $n \leq 3$, a contradiction,

- If $\Delta=4$, then $n<5$, a contradiction,

- If $\Delta=3$, then $n<9$, therefore $n=5$ or $n=7$.

In the case $n=5$, we must have a graph with five vertices, eight edges and $\Delta(G)=3$ which is impossible. If $n=7$, then $G$ is overfull and it is isomorphic with $\mathrm{C}_{7}+3 e$ and so,

Theorem 4. Among all graphs on $n$ vertices and $m=n+3$ edges, only $C_{7}+3 e$ is overfull.

In the following theorem the second extremal overfull graphs are computed:

Theorem 5. Let $n \geq 5$ be an integer, then

- The second maximal overfull graph on $n$ vertices is $K_{n}-e$,

- The second minimal overfull graph on $n$ vertices is $C_{n}+\frac{n-1}{2} e$.

Proof. By using Theorem 1, the proof of the first claim is clear. For the second part, note that $C_{n}+\frac{n-1}{2} e$ has a vertex of degree 2 and the others have degree 3 . So, by Euiler Theorem, we have:

$$
2 m=\sum_{v \in V(G)} \operatorname{deg}(v)=3(n-1)+2=3 n-1,
$$

thus,

$$
m=\frac{3 n-1}{2}>\frac{3}{2}(n-1) .
$$

This implies that $C_{n}+\frac{n-1}{2} e$ is overfull. On the other hand, $\frac{3 n-1}{2}-\frac{3}{2}(n-1)=1$. This means that $C_{n}+\frac{n-1}{2} e$ has the minimum possible edges by this properties and this completes the proof. 
To find the the third minimal overfull graph, note that the second minimal has $n-1$ vertices of degree 3 , so by adding a new edge to it we have $\Delta\left(C_{n}+\frac{n-1}{2} e\right)=4$ and so:

Theorem 6. Let $n \geq 5$ be an integer, then

- The third maximal overfull graph on five vertices is isomorphic with $C_{5}+2 e$.

- If $n>5$ then, the third maximal overfull graph on $n$ vertices is $K_{n}-2 e$,

- The third minimal overfull graph with $n \geq 7$ vertices is a graph constructed by removing an edge from a 4-regular graph.

Proof. The proofs of the first and second claims are trivial. For a minimal graph satisfies in the third condition, it is neccesary that $m-\frac{\Delta(n-1)}{2}=1$ and so, $m=2 n-1$. On the other hand, if $a, b$ be the number of vertices of degrees 3 and 4 , respectively, then $3 a+4 b=4 n-2$ and $a+b=n$. By solving these equations we find that $a=2$ and $b=n-2$. So, the third minimal graph has exactly two vertices of degree 3 and the others are degree 4 . It means that we can remove an edge from a 4-regular graph to obtain the third minimal.

Corollary 1. By the conditions of last theorem:

- The fourth maximal overfull graph on five vertices is isomorphic with $C_{5}$,

- For $n \geq 7$ the fourth maximal overfull graph is isomorphic with $K_{n}-3 e$,

- The fourth minimal overfull graph is a 4-regular graph on $n \geq 7$ vertices.

\section{Plannar Overfull Graphs}

In this section, we classify all plannar overfull graphs. To do this, we need followin lemma:

Lemma 4 [1]. If $G$ be a plannar graph on $n$ vertices and $m$ edges, then $m \leq 3 n-6$.

Theorem 7. Let $G$ be a plannar overfull graph, then

$$
G \cong C_{n} \text { or } G \cong C_{n}+\frac{n-1}{2} e .
$$

Proof. Since $G$ is plannar overfull graph, then

$$
\Delta \frac{n-1}{2}<m \leq 3 n-6 \text {. }
$$

This implies that $n<\frac{\Delta-12}{\Delta-6}$. Because $n \geq 3$, hence $\Delta \leq 3$ and we have the following cases:

- If $\Delta=2$, then $G \cong C_{n}$,

- If $\Delta=3$, then by Lemma 2, $G$ has no a pendant vertex. Let $a, b$ be the number of vertices of degrees 2 and 3, respectively. Thus, $2 a+3 b=2 m$ and $a+b=n$. Hence, $a=3 n-2 m$ and $b=2(m-n)$. Since $\Delta=3$ and $G$ is overfull graph, then $m>\frac{3}{2}(n-1)$ and so $a<3 n-3(n-1)=3$. Clearly, $b>n-3$ and we have the following cases:

Case 1. $a=1$, in this case $2 m=2+3(n-1)=3 n-1$ and therefore, $G \cong C_{n}+\frac{n-1}{2} e$.

Case 2. $a=2$, in this case, $b=n-2$ and then $2 m=2.2+3(n-2)=3 n-2$, a contradiction, since $2 m$ is an even integer, while $3 n-2$ is odd.

\section{REFERENCES}

[1] G. Chartrand and F. Zhang, "Chromatic Graph Theory," Chapman and Hall/CRC, London, 2008. doi:10.1201/9781584888017

[2] A. G. Chetwynd and A. J. W. Hilton, "Star Multigraphs with Three Vertices of Maximum Degree," Mathematical Proceedings of the Cambridge Philosophical Society, Vol. 100, No. 2, 1986, pp. 303-317. doi:10.1017/S030500410006610X

[3] T. Niessen, "How to Find Overfull Subgraphs in Graphs with Large Maximum Degree,” Discrete Applied Mathematics, Vol. 51, No. 1-2, 1994, pp. 117-125.

[4] M. Plantholt, "Overfull Conjecture for Graphs with High Minimum Degree,” Journal of Graph Theory, Vol. 47, No. 2, 2004, pp. 73-80. doi:10.1002/jgt.20013 